\title{
Material Modeling Approach for Graphene Antenna Design
}

\author{
Rajni Bala ${ }^{{ }^{1}}$, Anupma Marwaha ${ }^{2}$, Sanjay Marwaha ${ }^{3}$ \\ Sant Longowal Institute of Engineering \& Technology, \\ Longowal (Deemed University) Dist. Sangrur, Punjab, India \\ *Corresponding author, e-mail: rajnisliet@gmail.com¹, marwaha_anupma@yahoo.co.in², \\ marwaha_sanjay@yahoo.co.in ${ }^{3}$
}

\begin{abstract}
For the last few decades, in the field of patch antenna design for wireless applications the researchers have gone through experimenting with almost all types of variations in shape and dimensions of patch element, substrate material, exploiting with patch and ground structure with slots. Very little attention has been given to explore the pioneering approach of using different patch material improving the performance of antennas in terahertz regime. The graphene material is lately being used as patch material as it possesses a number of desirable electromagnetic and mechanical properties that assists in providing flexible and reconfigurable antenna structure. The material tunable conductivity accounts for the superior electromagnetic properties. Hence the authors have presented the simplified analytical solution for graphene surface conductivity to be utilized to characterize the graphene antenna parameters.
\end{abstract}

Keywords: graphene, tunable conductivity, nano patch antenna, terahertz regime, finite element method (FEM)

Copyright $(2015$ Institute of Advanced Engineering and Science. All rights reserved.

\section{Introduction}

Graphene's high electrical conductivity and high optical precision make it a candidate for transparent conducting electrodes, required for various high-speed electronics and high frequency applications due to its very high mobility and graphene's saturation velocity [1]. In particular, graphene's mechanical strength and flexibility are beneficial compared to indium tin oxide, which is brittle [2]. Single sheet of graphene are hard to make on a suitable substrate. So according to the radio wave absorption, stacked graphene layer on a silicon dioxide substrate increases the absorption of radio waves by $1.68 \%$ fractional bandwidth, extensible from microwave to terahertz frequencies, while remaining translucent to visible light.In present days the thrust is to mathematically model graphene-based plasmonic nano-antenna which can operate efficiently at micrometer radio wavelengths. Plasmonic propagation can be supported by graphene at $\mathrm{THz}$, leading to extremely interesting properties for practical antennas applications. The wavelength of surface plasmon polaritons for a given frequency is several hundred times smaller than the wavelength of freely propagating electromagnetic waves of the same frequency. The dimensions and speed differences allow efficient graphene-based antennas to be far smaller than conventional alternatives [3]. For using graphene as patch material major challenge is to mathematically model the new complex graphene material that would exhibit improved high frequency properties. The specific material properties that need improvement are lower losses, better impedance matching, high radiation efficiency and modified electromagnetic absorption characteristics compatible with nano design on the basis of electromagnetic properties. There has however been relatively little effort in improving the materials technology in antennas and simplifying mathematically the tunable graphene surface conductivity [4].

\section{Modeling Approach for Graphene Antenna Design}

In this paper an attempt has been made to accurately model graphene based material with tunable conductivity and hence the graphene based rectangular nano patch antenna has been designed operating in the terahertz $(\mathrm{THz})$ regime and good agreement has been observed 
as we compare the metallic antenna and much simpler graphene based nano patch antenna. From the previous literature it is known that the electromagnetic fields for metallic antenna are governed by classical Maxwell's equations, the graphene is however represented by a conductivity surface arising from a semiclassical intraband mode and quantum-dynamical interband mode [4]. The Kubo formula has therefore been used to calculate the graphene surface conductivity as a function of frequency. The surface conductivity of an infinite graphene film consists of two parts: first term is intraband and second term interband contributions in Equation (1), correspond to the intraband electron-phonon scattering process and interband electron transition respectively. In the infrared and visible range (short wavelength), the graphene optical conductivity is determined by interband transitions whereas, for terahertz range (long wavelengths) is dominated by intraband transitions. This simple conductivity model has been used here which ignores the magnetic field hall conductivity as graphene is assumed to be only electrically biased [5-6].

$$
\sigma\left(i \omega, \mu_{c}, \Gamma, \mathrm{T}\right)=-j \frac{q_{e}^{2}(\omega+2 j \Gamma)}{\prod \hbar^{2}}\left\lceil\frac{q_{e}^{2}}{(\omega+2 j \Gamma)^{2}} \int_{0}^{\infty} \varepsilon\left(\frac{\partial f_{d}(\varepsilon)}{\partial \varepsilon}-\frac{\partial f_{d}(-\varepsilon)}{\partial \varepsilon}\right) d \varepsilon-\int_{0}^{\infty} \frac{f_{d}(-\varepsilon)-f_{d}(\varepsilon)}{(\omega+2 j \Gamma)^{2}-\left(\frac{4 E}{h}\right)^{2}} d \varepsilon\right]
$$

Here $\omega$ is angular frequency of the photon, $\Gamma$ is electron scattering rate expressed in terms of relaxation time as $\tau=\frac{1}{2 \Gamma}, \hbar$ is the reduced Planck constant or Dirac constant i.e. $\hbar=\frac{h}{2 \Pi}$, where $\mathrm{h}$ is Planck constant, $\mathrm{T}$ is room temperature, the Fermi-dirac distribution function $f_{d}(\varepsilon)=\left(1+\frac{\varepsilon+\left|\mu_{c}\right|}{k_{B} T}\right)^{-1}$ and $\mathrm{k}_{\mathrm{B}}$ is the Boltzmann constant. It is noted that for highly doped or gated graphene $\frac{\mu_{C}}{k_{B} T}<1$, so the carrier density and chemical potential can be expressed as $n_{s}=\frac{\mu_{c}^{2}}{\pi \hbar^{2} V_{f}^{2}}$ and $\mu_{c} \approx \sqrt{\Pi \hbar^{2} v_{f}^{2} n_{s}}$ respectively. For present analysis when $\frac{\mu_{c}}{k_{B} T}>1$, therefore the chemical potential of graphene is determined in terms of carrier density from the expression given by:

$$
n_{s}=\frac{2}{\Pi \hbar^{2} V_{f}^{2}} \int_{0}^{\infty} \varepsilon\left[f_{d}\left(\varepsilon-\mu_{c}\right)-f_{d}\left(\varepsilon+\mu_{c}\right)\right] \partial \varepsilon
$$

For $\left|\mu_{c}\right|=\left\{\begin{array}{l}-\mu_{c}, \mu_{c}<0 \\ \mu_{c}, \mu_{c} \geq 0\end{array}\right.$ Now for the present case we consider $\mu_{C}>0 ; \mu_{C}$ is positive. For solving Equation (2), using substitution, $A=\int_{0}^{\infty} \varepsilon\left[f_{d}\left(\varepsilon-\mu_{c}\right)\right] \delta \varepsilon$ and $B=\int_{0}^{\infty} \varepsilon\left[f_{d}\left(\varepsilon+\mu_{c}\right)\right] \delta \varepsilon$.

The first Fermi-dirac distribution term can be expressed as:

$$
f_{d}\left(\varepsilon-\mu_{C}\right)=\frac{1}{\left(1+e^{\frac{\varepsilon-2 \mu_{C}}{k_{B} T}}\right)}
$$

Therefore,

$$
A=\int_{0}^{\infty} \varepsilon\left[\frac{1}{\left(1+e \frac{\varepsilon-2 \mu_{C}}{k_{B} T}\right)}\right] \partial \varepsilon
$$

Using the approximation $1+e^{\frac{\varepsilon-2 \mu_{C}}{k_{B} T}} \approx e^{\frac{\varepsilon-2 \mu_{c}}{k_{B} T}}$ Equation (4) reduces to:

$$
A=\int_{0}^{\infty} \varepsilon\left(e^{\frac{2 \mu_{c}-\varepsilon}{k B^{T}}}\right) \partial \varepsilon=e^{\frac{2 \mu_{c}}{k_{B} T}}\left[\int_{0}^{\infty} \varepsilon\left(e^{\frac{-\varepsilon}{k B^{T}}}\right) \partial \varepsilon\right]
$$


For further simplification, substitute $\frac{1}{k_{B} T}=\alpha, \alpha \varepsilon=y$ then $\alpha \partial \varepsilon=\partial y$ in Equation (4a),

$$
A=\frac{2 \mu_{c}}{k_{B} T}\left[\int_{0}^{\infty} \varepsilon\left(e^{-\alpha \varepsilon}\right) \partial \varepsilon\right]=e^{\frac{2 \mu_{C}}{k_{B} T}}\left[\int_{0}^{\infty} \frac{y}{\alpha}\left(e^{-y}\right) \frac{\partial y}{\alpha}\right]=e^{\frac{2 \mu_{C}}{k_{B} T}} \frac{1}{\alpha^{2}}\left[\int_{0}^{\infty} y\left(e^{-y}\right) \partial y\right]
$$

Solving this equation by using application of integration methods based on ILATE rule the simplified expression is obtained as:

$$
A=e^{\frac{2 \mu_{c}}{k_{B} T}}\left(k_{B} T\right)^{2}
$$

Similarly, the second term of carrier density expression given by $B=\int_{0}^{\infty} \varepsilon\left[f_{d}\left(\varepsilon+\mu_{c}\right)\right] \partial \varepsilon$ is solved correspondingly to obtain the solution as:

$$
B=\left(k_{B} T\right)^{2}
$$

Hence using the values of A and B in Eq. (2), the carrier density is obtained as:

$$
n_{s}=\frac{2}{\Pi \hbar^{2} V_{f}^{2}}\left(k_{B} T\right)^{2}\left(e \frac{2 \mu_{C}}{k_{B} T}-1\right)
$$

Where $V_{f}$ is the Fermi velocity $\left(\sim 3 \times 10^{6} \mathrm{~m} / \mathrm{s}\right.$ in graphene), $\varepsilon$ is the energy and $\mu_{c}$ is graphene chemical potential. The parameter of great interest for evaluating the performance of the graphene based nano devices are the chemical potential of graphene, i.e., the level in the distribution of electrons energies at which a quantum state is equally likely to be occupied or empty. The chemical potential $\mu_{c}$ can be accurately extracted by numerically solving Equation (6).

The graphene SPP can therfore be tuned by material doping which is further controlled by the external bias, $V_{D C}$. Hence the graphene conductivity $\sigma$ or correspondingly surface impedance $Z_{s}=1 / \sigma$ can be dynamically controlled by $V_{D C}$. This property can be used to create tunable nano devices with dynamic control. At the $\mathrm{THz}$ frequency region, the application of an external DC bias allows to increase chemical potential, thus reducing losses and increases the inductive behaviour of the graphene sheet.

The first term of conductivity represented by Equation (1) corresponds to intraband transition in which the real part contributes to energy absorption or dissipation due to the intraband electrons and after utilizing the solution explained above, can now be expressed as:

$$
\sigma(\text { int raband })=j \frac{q_{e}^{2} k_{B} T}{\Pi \hbar^{2}\left(\omega+j \tau^{-1}\right)}\left(\frac{\mu_{C}}{k_{B} T}+2 \ln \left(e^{\frac{-\mu_{C}}{k_{B} T}+1}\right)\right)
$$

In the recent years many researchers are using the Kubo's expression to find out tunability of graphene material but to the best of our knowledge very little concentration has been given to provide the accurate solution of the expression to analyze the effect of chemical potential on conductivity in simplified form [7-9]. Therefore the authors have suggested here an accurate mathematical modeling of graphene surface conductivity in a simple manner. The first attempt was performed using the Mathematica software, which however could not provide the converged solution. Thereafter MATLAB code was generated for the expression considering $\mu_{\mathrm{c}}$ $\neq 0$, and using the values of different parameters as $\mathrm{q}_{\mathrm{e}}=1.6 \times 10^{-19} \mathrm{C}$ with Coulomb charge $(\mathrm{C})=$ $6.25 \times 10^{19}$ electrons, $\mathrm{k}_{\mathrm{B}} \mathrm{T}=0.0256 \mathrm{eV}$ where $\mathrm{k}_{\mathrm{B}}$ is Boltzmann constant, $\hbar=6.582 \times 10^{-16} \mathrm{eVs}, \quad \Gamma$ $=0.11 \mathrm{eV}, \omega=2 \pi f$ where $\mathrm{f}$ is frequency.

$$
\sigma_{(\text {int raband })}=\frac{3.009 \mathrm{X}_{10} 10}{6.28 f+0.22 j}\left(\frac{\mu_{C}}{k_{B} T}+2 \ln \left(e^{\frac{-\mu_{c}}{k_{B} T}+1}\right)\right)
$$




$$
\begin{aligned}
& \sigma_{\text {int ra }(\text { realpart })}=\frac{0.66198 \mathrm{X}_{10} 0^{10}}{39.44 f^{2}+0.0484}\left(\frac{\mu_{C}}{0.0256}\right)+\frac{0.66198 \mathrm{X}_{10} 0^{10}}{39.44 f^{2}+0.0484}\left(2 \ln \left(e \frac{-\mu_{c}}{0.0256}+1\right)\right) \\
& \sigma_{\text {int ra }(\mathrm{i} \text { mg.part })}=j \frac{18.896 f \mathrm{X}_{10} 10}{39.44 f^{2}+0.0484}\left(\frac{\mu_{C}}{0.0256}\right)+\frac{18.896 f \mathrm{X}_{10} 10}{39.44 f^{2}+0.0484}\left(2 \ln \left(e^{\frac{-\mu_{c}}{0.0256}+1}\right)\right)
\end{aligned}
$$

Figure 1 and Figure 2 respectively show the real and imaginary part of intraband conductivity at terahertz frequencies regime obtained from their corresponding equations.

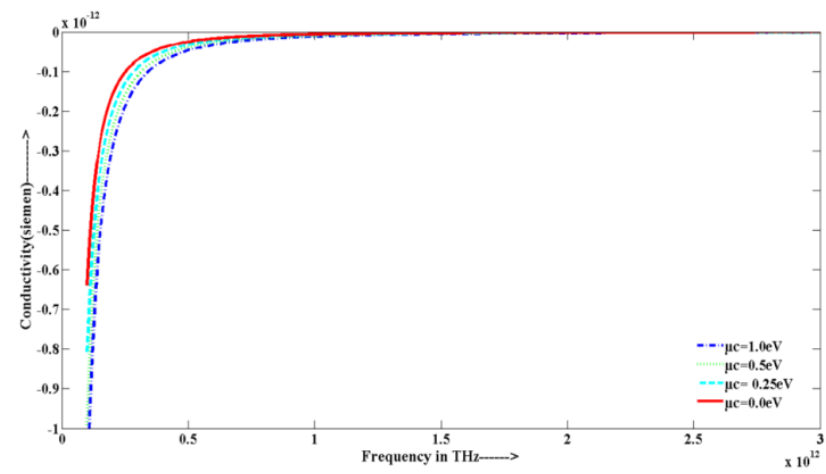

Figure 1. Real part of the intraband conductivity at room temperature $(T=300 \mathrm{~K})$ for different values of chemical potential

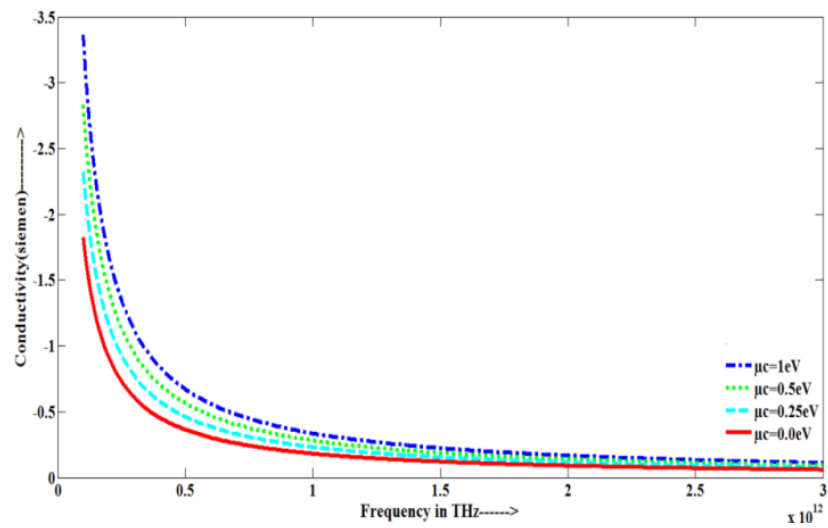

Figure 2. Imaginary part of the intraband conductivity at room temperature $(T=300 \mathrm{~K})$ for different values of chemical potential

Further Equatuion (7) be further simplified for $\mu_{c}=0$,

$$
\sigma(\text { int raband })=\frac{q_{e}^{2} k_{B^{T}}}{\Pi \hbar^{2}}(2 \ln 2)\left(\frac{j}{\left(\omega+j \tau^{-1}\right)}\right)=j \frac{262.06 f \mathrm{X}_{10} 0^{10}}{39.44 f^{2}+0.0484}
$$

So, for this case, it can be observed that we obtain the simplified intraband term of the graphene conductivity which is similar to the available Drude-like form [7], i.e.

$$
\left.\sigma_{\text {intra }} \text { simplified }\right)=j \frac{q_{e}^{2} \mu_{c}}{\Pi \hbar^{2}\left(\omega+j \tau^{-1}\right)}
$$




\section{Potential Application for Graphene Antenna Design}

For validation graphene based rectangular patch antenna has been designed and numerically analyzed. HFSS model is created as shown in Figure 3 with keeping the other dimensions of antenna model as given in Table 1. Fundamentally, the thickness of the dielectric substrate (t) should be less than the free space wavelength $\left(0.003 \lambda_{0} \leq h \leq 0.05 \lambda_{0}\right)$ [3]. The analysis is performed with graphene patch on silicon dioxide substrate material having thickness of $3 \mu \mathrm{m}$ and dielectric permittivity, $\varepsilon_{\mathrm{r}}=4$. The wave propagation velocity for graphene material depends on the patch dimensions, its resonant frequency and the Fermi energy of the structure. Following the concept, the resonant frequency of a graphene based antenna can be evaluated as $f_{o}=\frac{v_{f}}{2 L_{P}}$, where $v_{f}$ is the wave propagation velocity for graphene material [8] and $L_{P}$ stands for the nano patch length. Using the given patch dimensions the antenna resonant frequency is calculated as $3 \mathrm{THz}[10-12]$.

\section{Results and Analysis}

The proposed simplified formulation presented as above is used for analytically solving the conductivity expression. The graphene antenna is simulated and analyzed here considering zero chemical potential for graphene material for simplicity of implementation. The antenna is analyzed for return loss, gain, directivity, absorption cross-section and radiation efficiency.

Table 1. Dimensions of graphene based terahertz square patch antenna

\begin{tabular}{cc}
\hline Parameter & Value \\
\hline Operating frequency band $\left(\mathrm{f}_{\mathrm{o}}\right)$ & $2.63-2.74 \mathrm{THz}$ \\
Substrate length and width & $106 \mu \mathrm{m} \times 65 \mu \mathrm{m}$ \\
Substrate thickness $(\mathrm{h})$ & $3 \mu \mathrm{m}$ \\
Substrate dielectric constant $\left(\mathrm{SiO}_{2}\right.$, & 4.0 \\
$\left.\epsilon_{\mathrm{r}}\right)$ & $5 \mu \mathrm{m} \times 5 \mu \mathrm{m}$ \\
Side length and width of square patch & $10 \mathrm{~nm}$ \\
$(\mathrm{Lp} \times \mathrm{Wp})$ & $6.2 \mu \mathrm{m}$ \\
Patch height $(\Delta)$ & $23.8 \mu \mathrm{m}$ \\
Length of feed & $15 \mu \mathrm{m}$ \\
Width of feed & $1.6 \mu \mathrm{m}$ \\
Length of $\lambda / 4$ transformer &
\end{tabular}

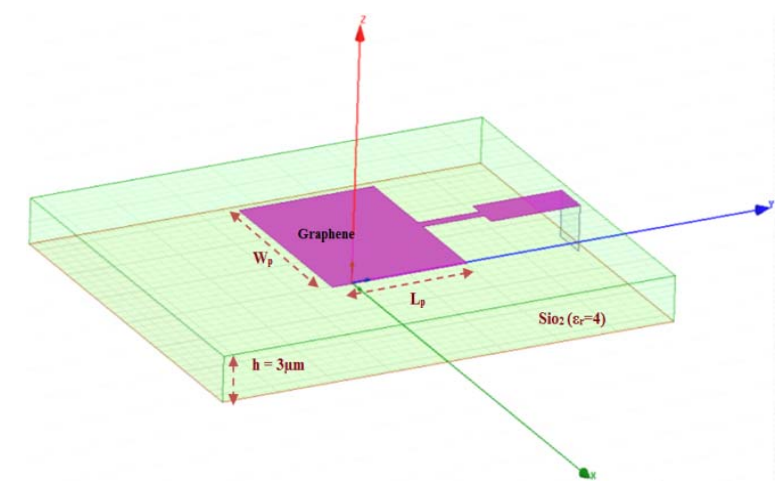

Figure 3. HFSS model of graphene based patch antenna

Figure 4 depicts, the return loss for the antenna with peak maximum value of $-21.09 \mathrm{~dB}$ at resonant frequency $2.70 \mathrm{THz}$. Figure 5 shows, the $2 \mathrm{D}$ plot of gain having maximum value of $6.6 \mathrm{~dB}$ at resonant frequency $2.70 \mathrm{THz}$. The gain apparently remains above $5 \mathrm{~dB}$ for the whole operating band of frequencies from 2.63-2.74 THz. 


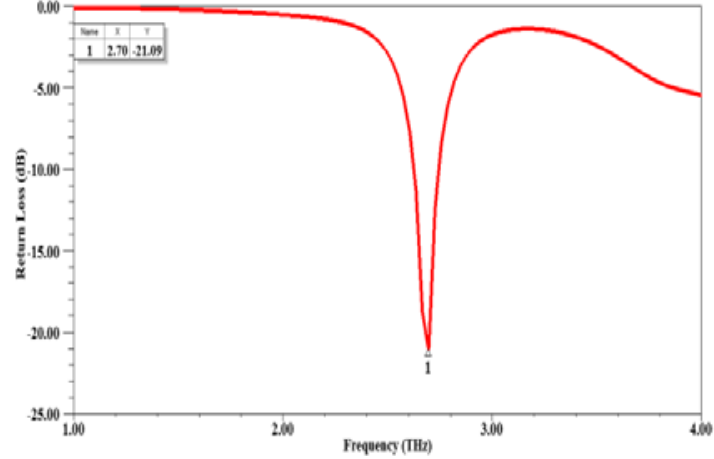

Figure 4. Return Loss (in dB) for graphene patch antenna

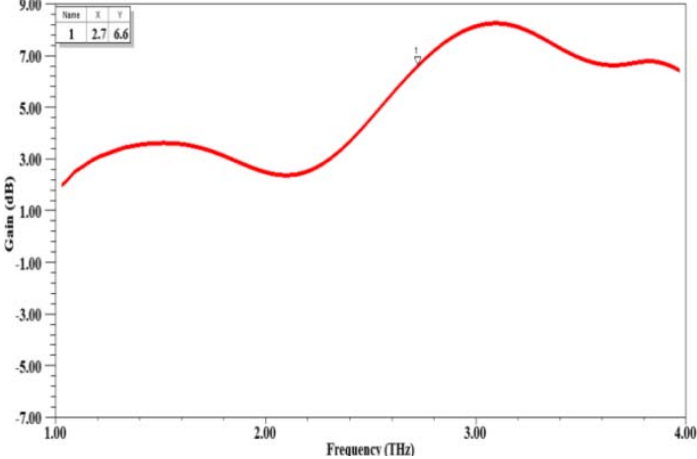

Figure 5. 2D gain plot (in $\mathrm{dB}$ )

The 3D radiation patterns for gain and directivity are as given in Figure 6 . Figure $7(a)$ demonstrates the 3D polar plot for $\mathrm{dB}$ gain in the azimuth plane $\phi=0^{\circ}$ (red) and $\phi=90^{\circ}$ (green).
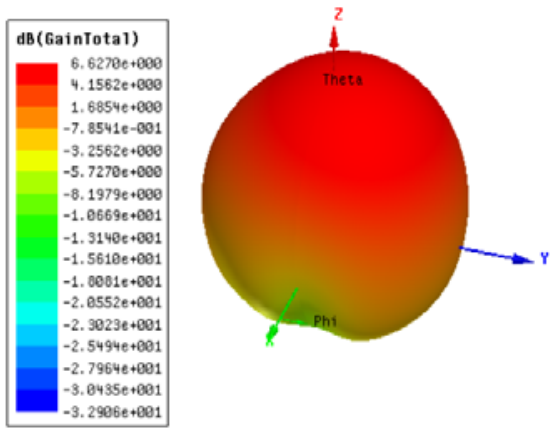

(a)
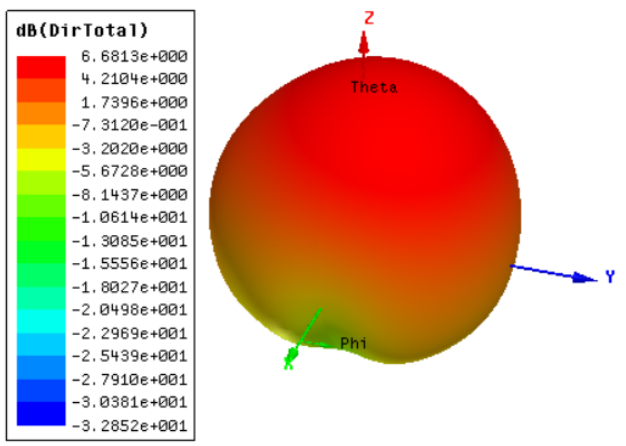

(b)

Figure 6. (a) Gain (in dB); (b) Directivity (in dB)

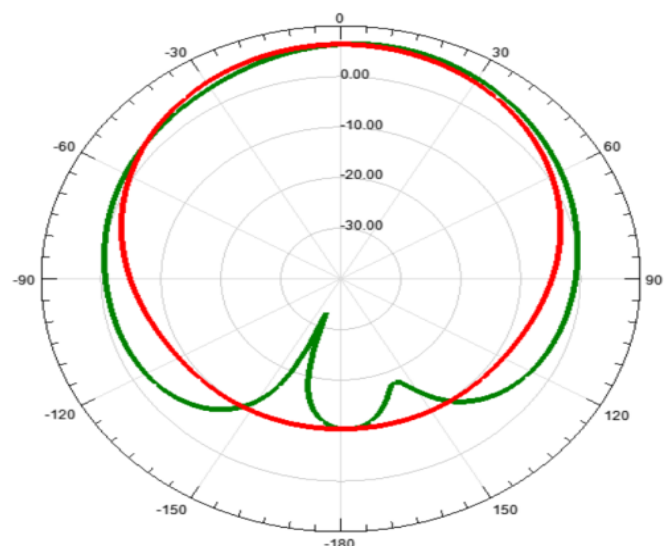

(a)

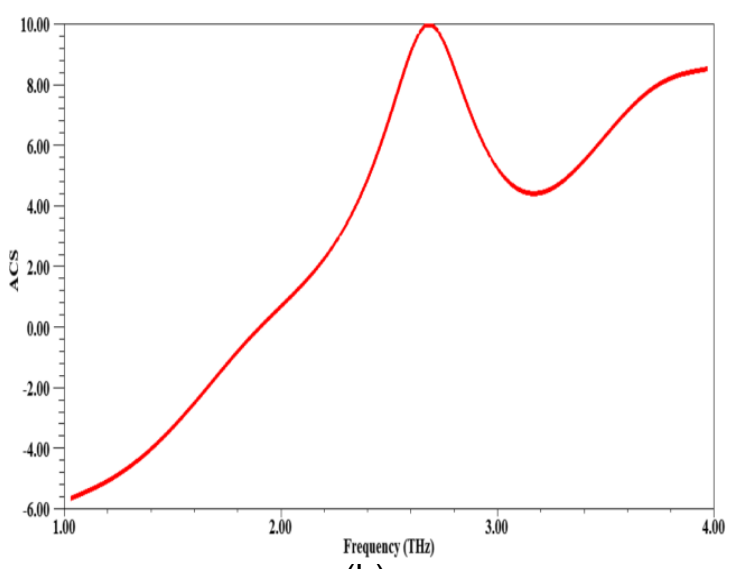

(b)

Figure 7. (a) 3D polar plot of dB gain in azimuth plane $\phi=0^{\circ}$ (red) and $\phi=90^{\circ}$ (green); (b) Absorption cross section rate 
The absoprtion coefficient is plotted as funcion of frequency in Figure 7 (b). The present analysis has been performed assuming graphene model with zero chemical potential hence resonating at a lower frequency with a small absorption cross section, which will limit their achievable radiation efficiency. On the other hand, graphene with a higher chemical potential posses greater absorption capabilities, but the resonant frequency also increases in that case, which may reduce their potential transmission range. The proposed graphene antenna therefore performs with the radiation efficiency of $88 \%$ at resonant frequency of $2.70 \mathrm{THz}$ as can be clearly seen from Figure 8.

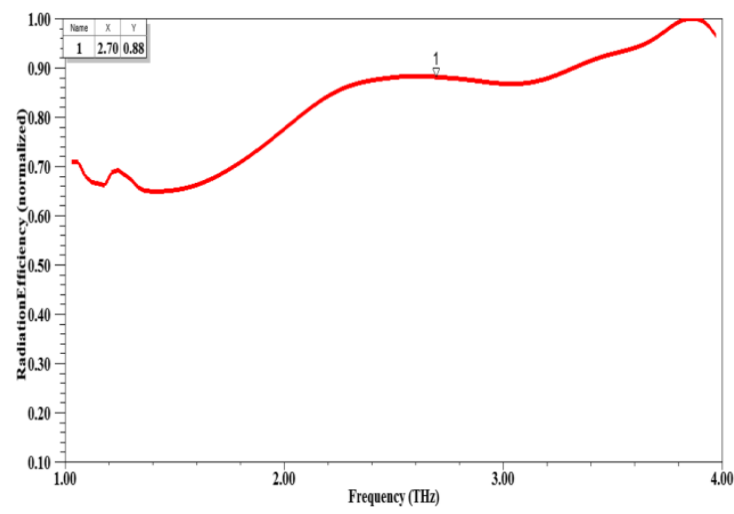

Figure 8. Radiation Efficiency

\section{Conclusion}

In this research paper simplified mathematical formulation for graphene material conductivity has been presented. The graphene based rectangular patch antenna on silicon dioxide substrate material has been designed thereafter to authenticate the validity of the proposed mathematical model. The simulation is numerically solved considering zero chemical potential for graphene material for simpler implementation on HFSS software. The antenna shows good impedance matching with $-21.09 \mathrm{~dB}$ return loss at resonant frequency of $2.70 \mathrm{THz}$. The with reasonably good gain of $6.627 \mathrm{~dB}$ and directivity of $6.6813 \mathrm{~dB}$ is achieved. The antenna radiates with maximum radiation efficiency over $88 \%$ at resonating frequency.

\section{Acknowledgements}

This work is supported by Department of Electronics and Communication Engineering of Sant Longowal Institute of Engineering and Technology, Longowal, Punjab, by providing excellent lab facilities such as High Frequency structural Simulator Software 13, Mathematica, MATLAB and other computational facilities.

\section{References}

[1] Wang H, Nezich D, Kong J, Palacios T. Graphene frequency multipliers. IEEE Electron Device Letters. 2009; 30(5).

[2] Meyer JC, Geim AK, Novoselov KS. The structure of suspended graphene sheets. Nature Publishing Group. 2007; 44: 60-63.

[3] Ming Lin Y, Jenkins KA, Farmer DB, Avouris P. Operation of graphene transistors at gigahertz frequencies. Nano Letters. 2009; 9(1): 422-426.

[4] Llatser I, Kremers C, Miquel J. Scattering of terahertz radiation on a graphene-based nano-antenna. American Institute of Physics Conference Proceedings. 2011; 1398: 144-146.

[5] Titz D, Bisognin A, Ferrero F, Laporte C, Ezzeddine H. $60 \mathrm{GHz}$ patch antenna using IPD technology. Loughborough Antennas and Propagation Conference (LAPC). Loughborough, UK. 2011.

[6] Atanassov Batovski D. Graphene and communications technology. Assumption University Journal of Technology. 2012; 16(2): 105-114.

[7] Gomez-Diaz JS, Perruisseau Carrier J. Microwave to $\mathrm{THz}$ properties of graphene and potential antenna applications. International Symposium on Antennas and Propagation. Nagoya, Japan. 2012.

TELKOMNIKA Vol. 16, No. 3, December 2015 : $480-487$ 
[8] Skulason HS, Nguyen HV, Guermoune A, Szkopek T. $110 \mathrm{GHz}$ measurement of large-area graphene integrated in low-loss microwave structures. Applied Physics Letter. 2011; 99(153504).

[9] Anand S, Sriram Kumar D, Jang Wu R, Chavali M. Graphene nanoribbon based terahertz antenna on polyimide substrate. Optik Science Direct. 2014; 125: 5546-5549.

[10] Gomez-Diaz JS, Perruisseau-Carrier J, Sharma P, lonescu AM. Non-contact characterization of graphene surface impedance at micro and millimeter waves. Journal of Applied Physics. 2012; 111: 114908-7.

[11] Huang Y, Wu L, Tang M, Mao J. Design of a beam reconfigurable thz antenna with graphene-based switchable high- impedance surface. IEEE Transactions on Nanotechnology. 2012; 11(4): 836-842.

[12] Morote ME, Sebastian J, Diaz G, Perruisseau Carrier J. Sinusoidally-modulated graphene leaky-wave antenna for electronic beamscanning at thz. Terahertz Science and Technology IEEE Transactions. 2014; 4(1): 116-122. 